\title{
ON A THEOREM OF HAAR
}

\author{
MASAAKI SHIBA
}

(Received December 20, 1968)

Let $\left\{\boldsymbol{\varphi}_{n}\right\}$ be a set of functions which are orthonormal on $[0,1]$. W. Rudin [3] (cf. A. Haar [1]) proved the following result.

Proposition. (I) If $\boldsymbol{\varphi}_{n} \in B V[0,1](n=1,2, \cdots)$, then $V\left(\varphi_{n}^{\prime}\right)>A n^{1 / 2}$, and

$$
\text { (II) if } \phi_{n} \in \Lambda_{1}[0,1] \quad(n=1,2, \cdots) \text {, then } N\left(\phi_{n}^{\prime \prime}\right)>A^{\prime} n \text {, }
$$

where $A$ and $A^{\prime}$ are positive constants, $V(f)$ is the total variation of $f$ $\in B V[0,1], N(f)=\sup _{x \neq y}|f(x)-f(y)| /|x-y|$ for $f \in \Lambda_{1}[0,1]$ and $\left\{\boldsymbol{\phi}_{n}^{\prime}\right\} \quad\left(\left\{\boldsymbol{\varphi}_{n}^{\prime \prime}\right\}\right)$ is a rearrangement of $\left\{\boldsymbol{\varphi}_{n}\right\}$ according to non-decreasing $V\left(\phi_{n}\right)\left(N\left(\boldsymbol{\varphi}_{n}\right)\right)$.

Recently, J. J. Price [2] showed (I) under the conditions $\sum_{m \neq n}\left|\left(\boldsymbol{\phi}_{m}, \boldsymbol{\phi}_{n}\right)\right|<\infty$ and $\left\|\varphi_{n}\right\|_{2}=1(n=1,2, \cdots)$ instead of $\left\{\boldsymbol{\varphi}_{n}\right\} \in O N[0,1]$.

In this note we extend W. Rudin's result under a much weaker hypotheses than those of J. J. Price.

Let $\left\{\boldsymbol{\varphi}_{n}\right\}$ be a set of functions in $L^{2}[0,1]$ such that

$$
\left\|\boldsymbol{\phi}_{n}\right\|_{2}=1 \quad(n=1,2, \cdots)
$$

and

$$
\sum_{m \neq n}\left|\left(\boldsymbol{\varphi}_{m}, \boldsymbol{\varphi}_{n}\right)\right|^{2}<\infty
$$

For $f \in \Lambda_{\alpha}^{p}[0,1](0<\alpha \leqq 1,1 \leqq p \leqq \infty)$ (cf. [4]) we put

$$
N_{\alpha}^{p}(f)=\sup \|f(\cdot+h)-f(\cdot)\|_{p} /|h|^{\alpha} .
$$

Then, we have the following. 
THEOREM. Under the conditions (1) and '2), if $\varphi_{n} \in \Lambda_{\alpha}^{p}[0,1](n=1,2, \cdots)$, then

(i) for $1 \leqq p \leqq 2$ and $1 \geqq \alpha>1 / p-1 / 2$,

$$
N_{\alpha}^{p}\left(\widetilde{\varphi}_{n}\right)>A n^{\alpha+1 / 2-1 / p}
$$

and

(ii) for $2 \leqq p \leqq \infty$ and $1 \geqq \alpha>0$,

$$
N_{\alpha}^{p}\left(\tilde{F}_{n}\right)>A^{\prime} n^{\alpha},
$$

where $A$ and $A^{\prime}$ are positive constants depending on the sum (2), $p$ and $\alpha$, and $\left\{\widetilde{\varphi}_{n}\right\}$ is a rearrangement of $\left\{\mathscr{\varphi}_{n}\right\}$ according to non-decreasing $N_{\alpha}^{p}\left(\phi_{n}\right)$.

(1) and (2) are satisfied for $\left\{\phi_{n}\right\} \in O N[0,1]$. So we have (I) from (i) and $N_{1}^{1}(f) \leqq A^{\prime \prime} V(f)$ for $f \in B V[0,1]$. We get (II) from (ii) for $p=\infty$ and $\alpha=1$. Further, we have the result of J. J. Price by (2) and (i).

Now, we need the following lemma.

LEMMA. If $f \in \Lambda_{\alpha}^{p}(-\pi, \pi)$ and $f$ is $2 \pi$-periodic, then

(i') for $1 \leqq p \leqq 2$ and $1 \geqq \alpha>1 / p-1 / 2$

$$
\left\|f-S_{n}(f)\right\|_{2}=O\left(n^{-(\alpha+1 \cdot 2-1, v}\right.
$$

and

(ii') for $2 \leqq p \leqq \infty$ and $1 \geqq \alpha>0$

$$
\left\|f-S_{n}(f)\right\|_{2}=O\left(n^{-\alpha}\right),
$$

where $f \sim \sum_{-\infty}^{\infty} \widehat{f}_{n} e^{i n x} \quad$ and $\quad S_{n}(f)=\sum_{|k| \leqq n} \widehat{f}_{k} e^{i k x}$.

Proof of Lemma. (i') We have

$$
f(x+h)-f(x-h) \sim 2 i \sum_{-\infty}^{\infty} \widehat{f}_{n} \sin (n h) e^{i n x} .
$$

From Hausdorff-Young's inequality we get 


$$
\begin{aligned}
\left(\sum_{-\infty}^{\infty}\left|\widehat{f}_{n} \sin (n h)\right|^{p^{\prime}}\right)^{1 / p^{\prime}} & \leqq A_{p}\|f(\cdot+h)-f(\cdot-h)\|_{p} \\
& =O\left(h^{\alpha}\right) \text { for } 1 \leqq p \leqq 2
\end{aligned}
$$

where $1 / p+1 / p^{\prime}=1$. Putting $h=\pi / 2^{v+1}$, then

$$
\begin{aligned}
\text { const. }\left(\sum_{2^{\nu-1}<|n| \leqq 2^{\nu}}\left|\widehat{f}_{n}\right|^{p^{\prime}}\right)^{1 / p^{\prime}} & \leqq\left(\sum_{2^{\nu-1<|n| \leqq 2^{\nu}}}\left|\widehat{f}_{n} \sin (n h)\right|^{p^{\prime}}\right)^{1 / p^{\prime}} \\
& =O\left(2^{-\nu \alpha}\right) .
\end{aligned}
$$

For $2^{\mu-1}<|n| \leqq 2^{\mu}$,

$$
\begin{aligned}
\left\|f-S_{n}(f)\right\|_{2}{ }^{2} & \leqq \sum_{|n|>2^{\mu}}\left|\widehat{f}_{n}\right|^{2}=\sum_{\nu=\mu}^{\infty} \sum_{2^{\nu-1}<|n| \leqq 2^{\nu}}\left|\widehat{f}_{n}\right|^{2} \\
& \leqq \sum_{\nu=\mu}^{\infty}\left(\sum_{2^{\nu-1}<|n| \leqq 2^{\nu}}\left|\widehat{f}_{n}\right|^{p^{\prime}}\right)^{2 / p^{\prime}} \cdot 2^{\nu(2-p) / p} \\
& \leqq B_{p, \alpha} \sum_{\nu=\mu}^{\infty} 2^{-\nu(2 \alpha+1-2 / p)}
\end{aligned}
$$

From $2 x+1>2 / p$, we have

$$
\left\|f-S_{n}(f)\right\|_{2}=O\left(2^{-\mu(\alpha+1 / 2-1 / p)}\right)=O\left(n^{-(\alpha+1 / 2-1 / p)}\right) .
$$

(ii') $\Lambda_{\alpha}^{p} \subset \Lambda_{\alpha}^{2}$ for $p \geqq 2$, so that by (i')

$$
\left\|f-S_{n}(f)\right\|_{2}=O\left(n^{-\alpha}\right) \quad \text { for } \quad f \in \Lambda_{\alpha}^{p} \quad(p \geqq 2) .
$$

This is the best possible, because (cf. A. Zygmund [ 4 ])

$$
f=\sum_{n=1}^{\infty} e^{i c n(\log n)} e^{i n x} / n^{1 / 2+\alpha} \in \Lambda_{\alpha}(0<\alpha<1)
$$

but

$$
\left\|f-S_{n}(f)\right\|_{2}^{2}>A \sum_{k>n} 1 / k^{1+2 \alpha} \sim n^{-2 \alpha}
$$


Proof of Theorem. From (1) and (2), we have easily the following

$$
\sum_{k=1}^{n} c_{k}{ }^{2} \leqq M\|f\|_{2}^{2} \text { for } f \in L^{2}[0,1] \text { and } c_{k}=\left(f, \varphi_{k}\right),
$$

where $M$ is a positive constant depending on the sum (2). Let $\left\{\psi_{k}\right\}$ be a cosine set on $[0,1]$ and we put

$$
\lambda_{n}^{p, \alpha}=\sup _{0<N_{\alpha}^{p}(f)<\infty}\left\|f-S_{n}(f)\right\|_{2} / N_{\alpha}^{p}(f) \text { for } f \in \Lambda_{\alpha}^{p},
$$

where $f \sim \sum \widehat{f}_{n} \psi_{n}, S_{n}(f)=\sum_{k=0}^{n} \widehat{f}_{k} \psi_{k}$ and $\widehat{f}_{n}=\left(f, \psi_{n}\right)$. From the lemma, we have

(6) $\quad \lambda_{n}^{p, \alpha}=\left\{\begin{array}{l}O\left(n^{-(\alpha+1 / 2-1 / p)}\right) \text { for } 1 \leqq p \leqq 2 \text { and } 1 \geqq \alpha \geqq 1 / p-1 / 2 \\ O\left(n^{-\alpha}\right) \text { for } p \geqq 2 \text { and } 1 \geqq \alpha>0 .\end{array}\right.$

It follows from the definition of $\lambda_{n}^{p, \alpha}$ that

$$
\sum_{i \geqq n+1}\left(\phi_{k}, \Psi_{i}\right)^{2} \leqq\left(\lambda_{n}^{p, \alpha} N_{\alpha}^{p}\left(\phi_{k}\right)\right)^{2}
$$

Since $\left\{\psi_{n}\right\}$ is a complete set,

$$
1=\sum_{i=1}^{\infty}\left(\boldsymbol{\phi}_{k}, \psi_{i}\right)^{2} \leqq\left(\lambda_{n}^{p, \alpha} N_{\alpha}^{p}\left(\phi_{k}\right)\right)^{2}+\sum_{i=1}^{n}\left(\phi_{k}, \psi_{i}\right)^{2}
$$

Adding these inequalities for $k=1,2, \cdots, m$ and applying (5), we get

$$
m \leqq\left(\lambda_{n}^{p, \alpha}\right)^{2} \sum_{k=1}^{m}\left(N_{\alpha}^{p}\left(\boldsymbol{\varphi}_{k}\right)\right)^{2}+M \cdot n \quad \text { for every } m \text { and } n .
$$

This inequality holds for $N_{\alpha}^{p}\left(\widetilde{\boldsymbol{\varphi}}_{k}\right)$, so putting $n=[m / 2 M]$,

$$
m / 2 \leqq\left(\lambda_{n}^{p, \alpha} N_{\alpha}^{p}\left(\widetilde{\varphi}_{m}\right)\right)^{2} \cdot m
$$

From (6) we have the results. 


\section{REFERENCES}

[1] A. HAAR, Über einige Eigenschaften der orthogonalen Funktionen-systeme, Math. Zeit., 31(1930), 128-137.

[2] J. J. Price, On a theorem of Haar, Proc. Amer. Math.Soc., 18(1967), 1056-1057.

[3] W.RUDIN, $L^{2}$-approximation by partial sums of orthogonal developments, Duke Math. Journ., 19(1952), 1-4.

[4] A.ZYGmUND, Trigonometric series I, Cambridge Univ. Press, 1959.

DEPARTMENT OF MATHEMATICS

FUKUSHIMA UNIVERSITY

FUKUSHIMA, JAPAN 